\title{
APPLICATION OF MACHINE LEARNING FOR REMOTE ELECTRONICS EXPERIMENTS AS THE MEAN OF INDENTIFICATION
}

\author{
Antoni Kozic \\ Vilniaus kolegija/University of Applied Sciences, Lithuania \\ Andzej Lucun \\ Vilniaus kolegija/University of Applied Sciences, Lithuania \\ Mindaugas Vingelis \\ LUMO Advanced Lighting Company Expert, Lithuania \\ Eugenijus Macerauskas \\ Vilniaus kolegija/University of Applied Sciences, Lithuania \\ Audrius Narmontas \\ Vilniaus kolegija/University of Applied Sciences, Lithuania
}

\begin{abstract}
We are increasingly faced with automated solutions that create a dynamic and constantly evolving scientific and technical environment, with new challenges in finding more accurate and efficient solutions for identification. This article introduces a machine learning application that allows you to automatically recognize and identify learners in a distance learning experiment without being explicitly programmed. Experimental methods and components have been transferred to a distance learning laboratory environment. A conceptual model of an electronics laboratory has been developed for remote experiments using identification methods. This article focuses on machine learning using the LabVIEW package, which can access data and use it for self-study. The article presents the application of machine learning software LabVIEW National Instruments and NI ELVIS hardware simulators for electronic laboratory remote experiments and perspectives in the educational process. Keywords: machine learning; LabVIEW; remote laboratory.
\end{abstract}

\section{Introduction}

The purpose of this article is to explore the potential of learning technologies and show how laboratories can develop a new level of quality. The current trend is to use measurement tools developed using virtual device technology for educational purposes (Ertugrul, 2000). Sources of recent research emphasize that there is an increasing need for learning that allows students to construct knowledge themselves through vigorous activity, and not just participate in the transfer of information. Practice shows that it is best to retain knowledge based 
Kozic et al., 2021. Application of Machine Learning for Remote Electronics Experiments as the Mean of Indentification

on personal work experience. A source of such experience can be both theoretical and practical experiments conducted in the laboratory of electronics. There is a lack of tools that allow students to solve problems, problems, or observe a phenomenon through experimentation. The software in this software environment is developed visually and does not require detailed programming knowledge from the developer. Electronic experiment is seen as a component of blended learning the environment, where you can learn from remote workplaces, independently of carrying out laboratory work. Experimentation becomes an instrument in which knowledge is acquired by experiments. It should be possible to perform them independently, for which mechanisms of control of identification and by means of machine learning are introduced. The aim of the study is to create a object of identification that identifies a student in remote electronics experiments. Machine learning algorithms are used to record the automatic identification process. The machine learning program that allows you to automatically recognize the students in the experiment in distance learning. In case of incorrect identification, fuses are introduced to obtain stop frame of the video. We use standard LabVIEW/ Analytics and Machine Learning Toolkit machine learning methods for identification. It is a software add-on for LabVIEW that provides training machine learning models. You can use these models to discover patterns in large amounts of data with anomaly detection and classification, and clustering algorithms. Additionally, these models can recognize patterns in new data. Learning, like artificial intelligence, covers such a broad range of processes that it is difficult to define precisely. Machine learning usually refers to the changes in systems that perform tasks associated with artificial intelligence (AI) such tasks involve planning, recognition, control, diagnosis, prediction, etc. The changes might be either enhancements to already performing systems or non-empirical synthesis of new systems. Different learning mechanisms might be employed depending on which subsystem is being changed.

\section{Methodology}

Now this is a hot topic that is widely discussed all over the world. The E-lab is perceived as a component of blended learning - an environment where you can study from remote workplaces, independently carry out laboratory work (Bonk, Graham, 2012). Lack of electronic tools that would allow students to solve problems, problems or observe a phenomenon, through experiments, input or selection of input data. The application of the experimental method in modern distance learning systems is only one of the important reasons for the development of remote electronics laboratories for practical and laboratory work, which is especially important now because of the COVID-19 pandemic (Gamage et al., 2020). The biggest benefit of such a remote experiment is that the student can 
access the experiment anytime, anywhere. If the student has to conduct the experiment on his or her own, an identification problem arises, or he or she performs the experiment on his or her own. We need to create a plug-in to the experiment, which will allow to identify the student. We are faced with automated solutions, new challenges in the search for more accurate and efficient solutions. These tasks require deeper theoretical and practical knowledge, special equipment and artificial intelligence algorithms. Using machine learning, we can automate the identification process by receiving reports or a student independently performing an experiment using image identification via a webcam using machine learning (Relf, 2003). The structure of the electronics experiment system presented in Figure 1.

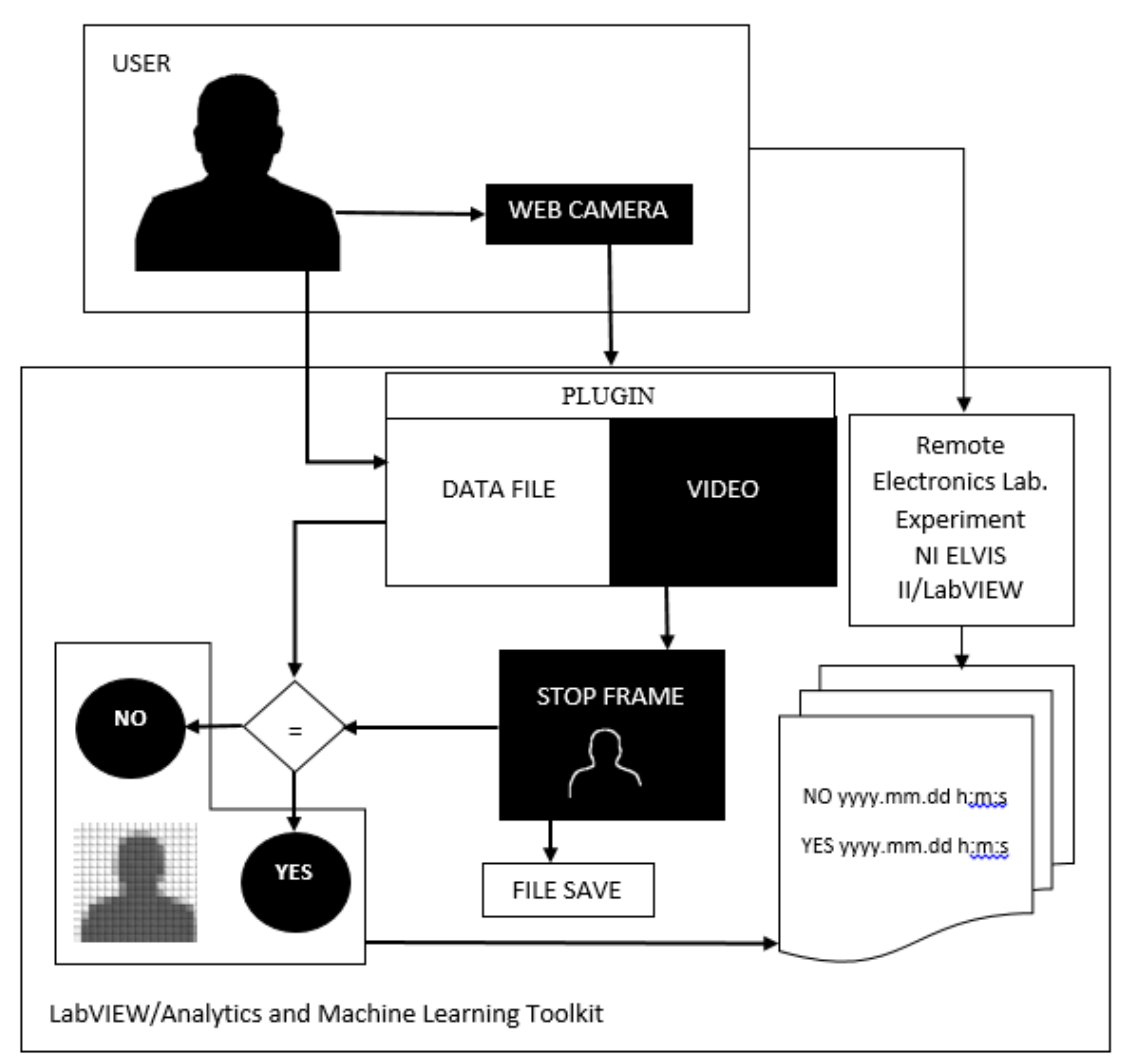

Figure 1 Block Diagram for the System

The basic idea is to create an identity using devices and software packages that can help implement this control. Application of artificial intelligence (AI) that provides systems the ability to automatically learn and improve from experience without being explicitly programmed (Nguyen et al., 2019) Machine learning focuses on the development of computer programs that can access data and use it learn for themselves. It is based on the universal training device NI ELVIS II (National Instruments Educational Laboratory Virtual Instrumentation suite), 
Kozic et al., 2021. Application of Machine Learning for Remote Electronics Experiments as the Mean of Indentification

which is used in conjunction with LabVIEW (Laboratory Virtual Instrumentation Engineering Workbench) and specialized instruments Analytics and Machine Learning Toolkit (National Instruments Corporation, 2018). NI ELVIS II also allows full automation of reporting over a computer network, and security measures prevent duplication. A key novelty is to uncover the potential of teaching technologies to provide a level of quality for classical electronics laboratory experiments (Ursutiu, Cotfas, Samoila, Zamfira \& Auer, 2004).

The NI ELVIS II connects through a mounting plate that connects the experimental electronics circuitry through the appropriate data and power inputs (Borodin, Batovrin \& Romanov, 2009).

The operation of the system is illustrated in Figure 2.

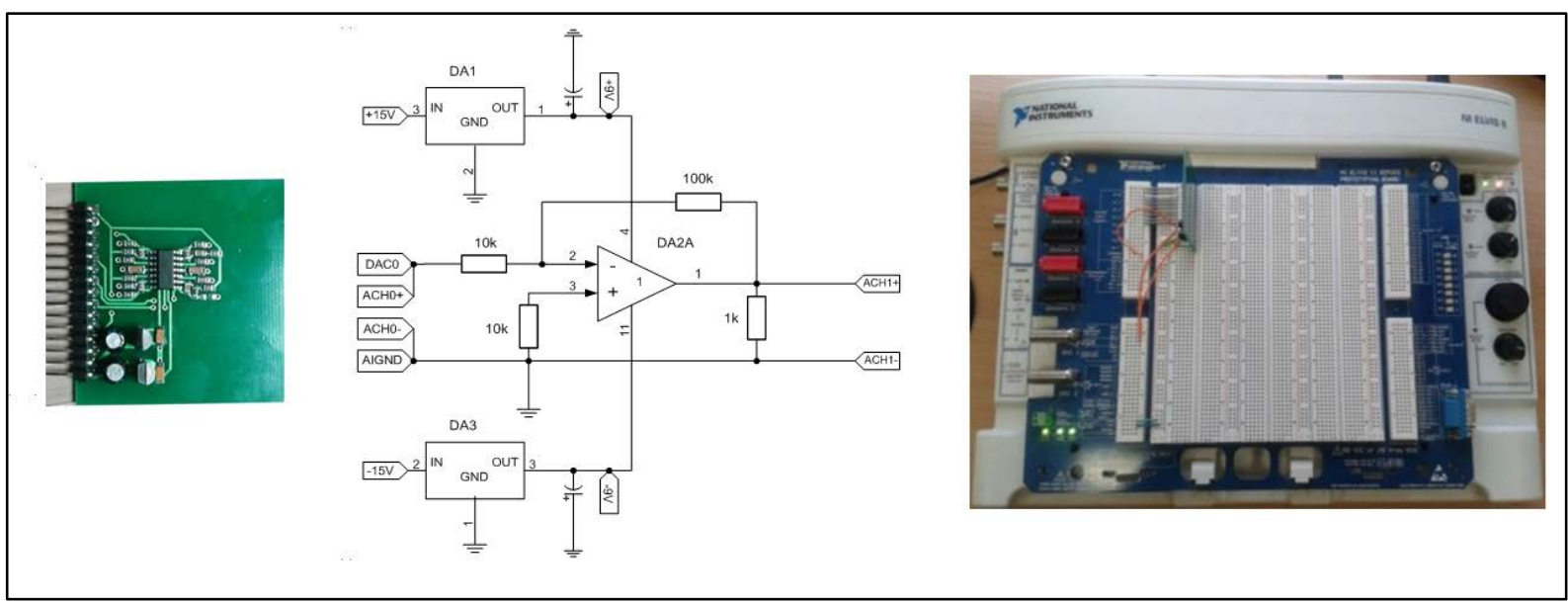

Figure 2 The System of the Electronics Experiments

A significant novelty is that this is not a virtual laboratory based on the software, but a real experiment with a remote-controlled electronics. The developed simplified conceptual model of the electronics laboratory is suitable for remote experimentation and demonstration (Kozic, Macerauskas \& Sakalys, 2016).

\section{Research Results}

The use of the advanced NI ELVIS II ensures the modernity of teaching methods and acquired knowledge. The LabVIEW package allows you to create a remote control system that allows you to remotely control the front panel of a program, a circuit connected to the NI ELVIS II device, using a web browser. The essence of the whole idea is to create a remote control that can be connected to an NI ELVIS II device connected to a server computer, which will allow you to perform the assigned tasks. This login option will allow the customer to log in 
from anywhere and perform specified tasks using their web browser. This is an excellent example of the development of modern technology that allows you to achieve excellent results. This ability to connect to tasks allows you to choose a location, saving time and space. Using NI ELVIS II and LabVIEW training tools, electronic experiments can be performed remotely and allow full automation of reporting over a computer network, while using identification tools allows writeoffs to be avoided.
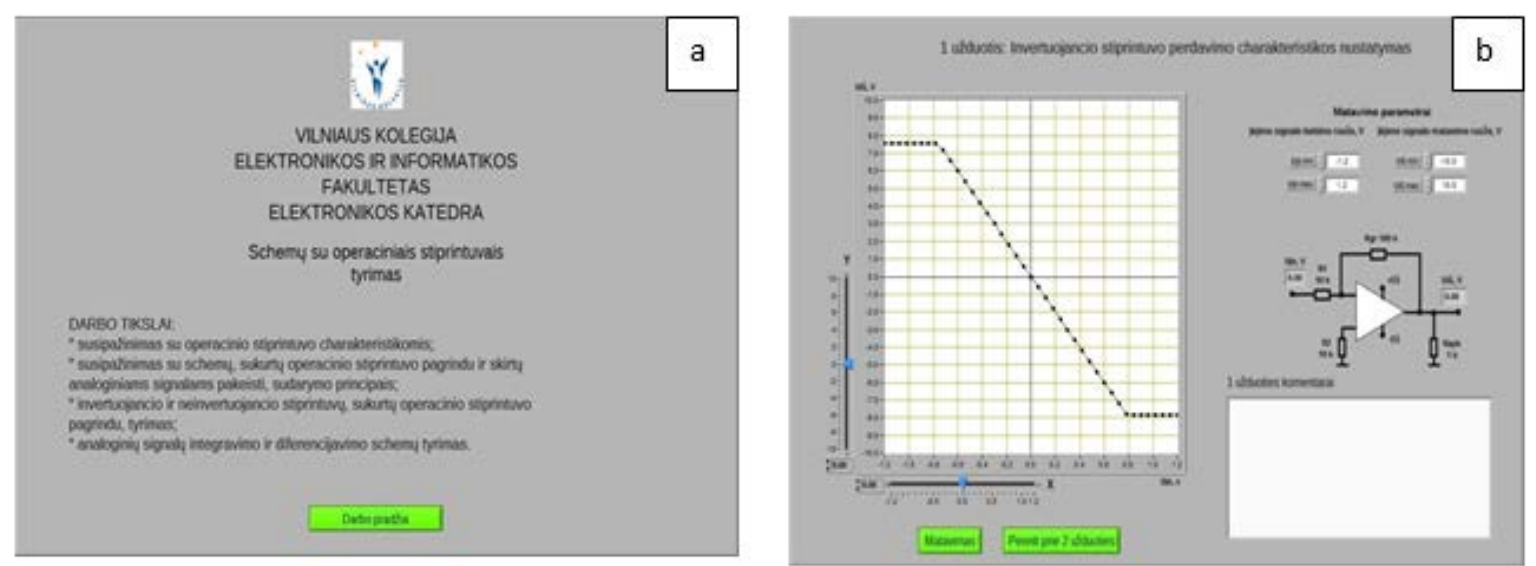

Figure 3 Primary Electronics Experiment Task Window (a) and Primary Task Pages - LabVIEW

The LabVIEW package allows you to create a remote control system that allows you to remotely control the front panel of a program, a circuit connected to the NI ELVIS II device, using a web browser. Remote control is based on local network use via VPN with identification codes issued to students who first connect to the VPN and then to the task. To control the electronics experiment via a browser, you must first save the application data to the server computer. To do this, use the LabVIEW function of the Web Publishing Tool. The generated web address is recorded in the web browser of the students that has the Run-Time Engine add-in installed. After saving, an image of the experiment appears. What changes will be made to the interface window displayed in the client browser will simultaneously occur on the server computer in the LabVIEW interface window (Salzmann, Gillet \& Huguenin, 2000). In other words, a virtual tunnel is created. By connecting a local IP address to an external one, you can connect in the router settings even outside the local network. It is also possible to track who connected to the server, which traffic data is at work, if you want clients/students that can be stopped or deleted (see. Figure 4). 
Kozic et al., 2021. Application of Machine Learning for Remote Electronics Experiments as the Mean of Indentification

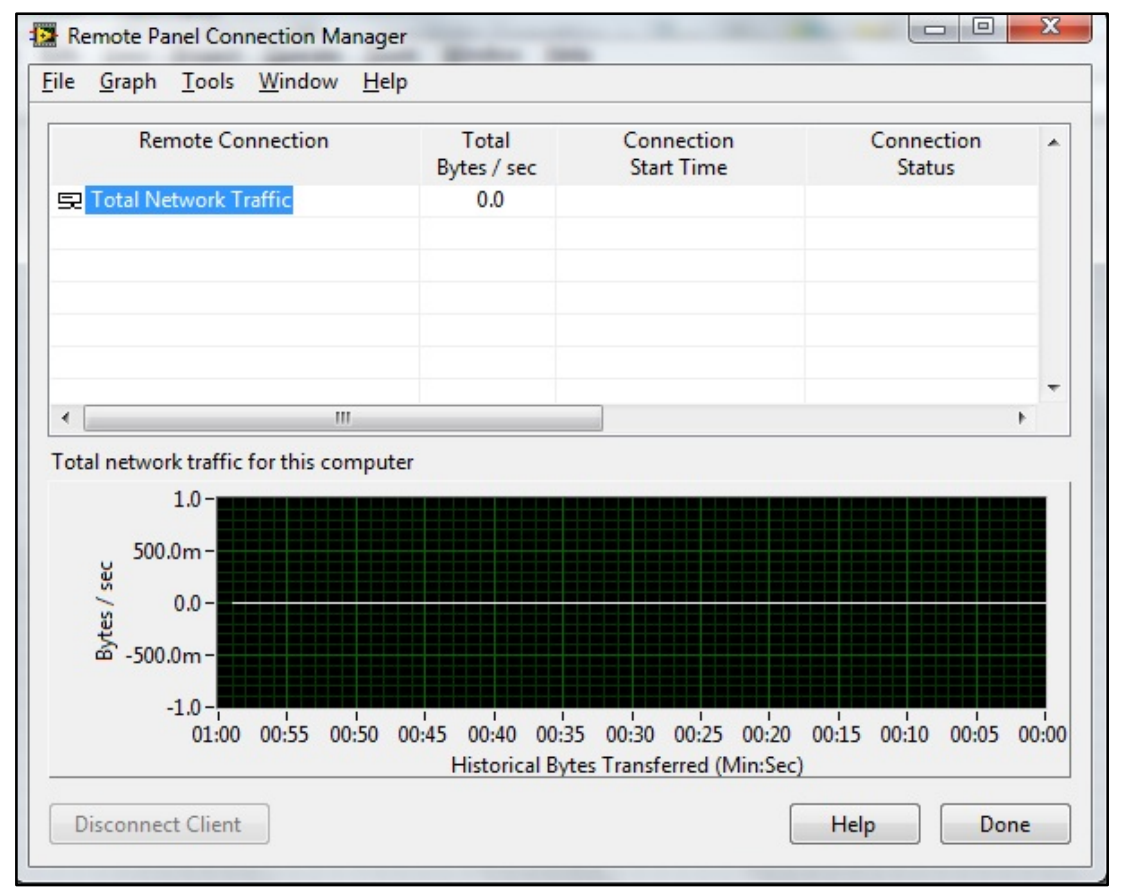

Figure 4 Traffic Monitoring and Control Window

In this window (see Fig. 4) you can see when the work started, what is the status of the connection and data traffic visually. This is a handy tool that is very useful when more than one customer is logged in, or when several want to log in. From the server side, you can control who is connecting and you can see who is waiting in your queue to connect.

While this is a great system for expanding standard learning opportunities. It, like all systems, has its drawbacks. Students can log into the system with their own identification codes, but others can complete the assignments and control of the system through the Remote Panel Connection Manager is not possible for 24 hours.

Thus, the goal is to use the principles of automation to monitor and train the system to identify students using machine learning algorithms (LabVIEW/ Analytics and Machine Learning Toolkit), therefore a plugin is used (see Fig. 5).

Shortcomings were found after the experiment was performed and the inaccurate results of identification were received. False messages are believed to be received due to identification mismatch using standard machine learning algorithms using LabVIEW/ Analytics and Machine Learning Toolkit. Therefore, the plugin has a stop frame every time. Thus, you can always view the file and, in case of doubt, determine whether the assignment was completed by the student. 


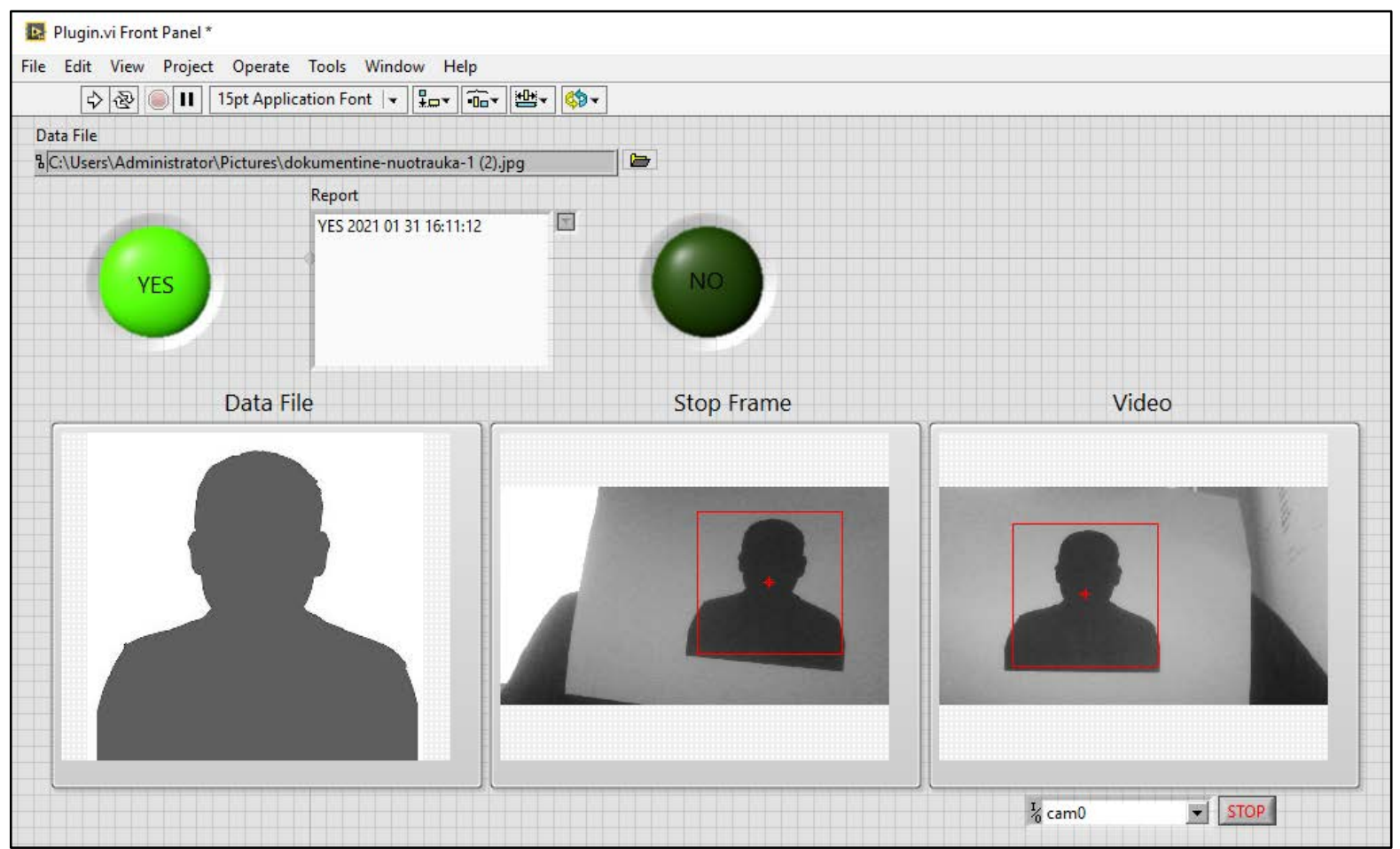

Figure 5 Example Identification via Plug LabVIEW

\section{Conclusions}

The essence of the experiment is that the use of LabVIEW/NI ELVIS technologies allows you to easily create any measuring complexes, adapt them to training programs. In terms of enhancing the quality of teaching, the biggest benefit of such an experiment is that the student can access the lab anytime, anywhere. Despite the fact that students log in with their own code, they are constantly identified or the task is completed independently. Application of machine learning for remote electronics experiments as a means of identification, the following conclusions can be made:

- Identifying the student for laboratory electronics experiments makes it easier to identify the student doing the assignment on his own.

- Algorithms of machine learning LabVIEW allow you to capture and control the process automatically, which would be difficult for a person for 24 hours.

- Performing such remote electronics experiments increases student motivation because tasks can be done remotely. 
Kozic et al., 2021. Application of Machine Learning for Remote Electronics Experiments as the Mean of Indentification

\section{References}

Bonk, C.J., Graham, C.R. (2012). The Handbook of Blended Learning-Global Perspectives, Local Designs, Wiley Published. ISBN 978-0-7879-7758-0.

Borodin, S., Batovrin, V. \& Romanov, A. (2009). LabVIEW Elektronikos praktiniai darbai (89-112). Learning materials: VPU Press. ISBN 978-9955-20-480-0.

Ertugrul, N. (2000). Towards virtual laboratories: A survey of LabVIEW-based teaching/learning tools and future trends. International Journal of Engineering Education, 16(3), 171-180.

Gamage, K.A.A., Wijesuriya, D.I., Ekanayake, S.Y., Rennie, A.E.W., Lambert, C.G., \& Gunawardhana, N. (2020). Online Delivery of Teaching and Laboratory Practices: Continuity of University Programmes during COVID-19 Pandemic. Education Sciences, 10(10), 291. DOI: 10.3390/educsci10100291

Kozic, A., Macerauskas, E., \& Sakalys, P. (2016, May). Remote laboratory as conceptual model of blended learning. In SOCIETY. INTEGRATION. EDUCATION. Proceedings of the International Scientific Conference, Vol. 2, 549-557.

National Instruments Corporation. (2018). LabVIEW 2018 Analytics and Machine Learning Toolkit Readme, NI 2009. Retrieved from https://www.ni.com/pdf/ manuals/377061b.html

Nguyen, G., Dlugolinsky, S., Bobak, M., Tran, V., Garcia, Á.L., Heredia, I. \& Hluchy, L. (2019). Machine learning and deep learning frameworks and libraries for large-scale data mining: a survey. Artificial Intelligence Review, 52(1), 77-124.

Relf, C.G. (2003). Image acquisition and processing with LabVIEW. CRC press.

Salzmann, C., Gillet, D., \& Huguenin, P. (2000). Introduction to real-time control using LabVIEW with an application to distance learning. Int. J. Engng Ed, 16(5), 372-384.

Ursutiu, D., Cotfas, P., Samoila, C., Zamfira, S. \& Auer M. (2004). NI-ELVIS in Remote Electronic Laboratory REL. Proceedings of the $1^{\text {st }}$ International Symposium on Remote Engineering and Virtual Instrumentation, Villach, Austria, 28. / 29. September 2004. ISBN3-89958-090-7. 